\title{
A Rare Case of Spontaneous Pneumopericardium
}

\author{
Abdallah Almaghraby ${ }^{1 *}$, Ahmed Samoka ${ }^{1}$, Mahmoud Shehata ${ }^{2}$, Hoda Zahran ${ }^{2}$, Yehia Saleh ${ }^{1}$, and Basma \\ Hammad $^{3}$ \\ ${ }^{1}$ Department of Cardiology, University of Alexandria, Egypt \\ ${ }^{2}$ Department of Cardiac, Thoracic and Vascular Sciences, University of Padova, Italy \\ ${ }^{3}$ Massachusetts General Hospital, Boston, USA
}

Received: April 11, 2017 Published: May 16, 2017

*Corresponding author: Abdallah Almaghraby, Department of Cardiology, University of Alexandria, Egypt

\section{Introduction}

Pneumopericardium is defined as a collection of air or gas in the pericardial cavity, its causes are usually secondary to trauma or mechanical ventilation, but spontaneous cases are very rare [1].

\section{Case Report}

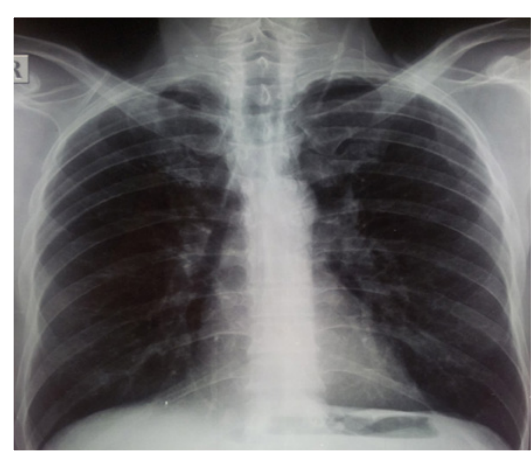

Figure 1: Plain chest $x$-ray showing the collection of air under the parietal pericardial layer.

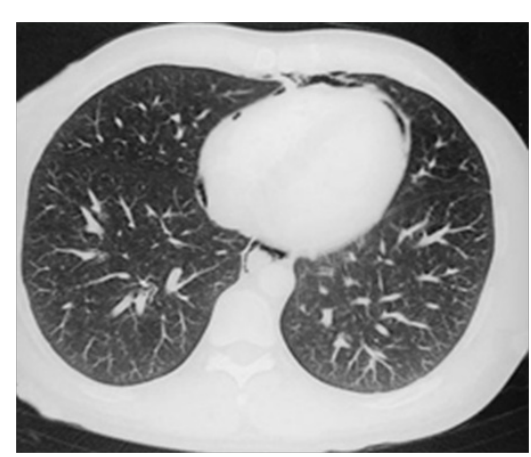

Figure 2: Plain chest computed tomography showing the collection of air under the parietal pericardial layer.

A 54-year-old man presented with a 6-hour history of acute typical retrosternal chest pain that occurred during minor exertion and was persistent from the start and not relieved by analgesics. His medical history was unremarkable, he was a heavy smoker. His clinical examination was unremarkable. His electrocardiogram (ECG) showed ST segment elevation in the inferior leads with PR depression in the anterior leads. His chest X-ray (CXR) leads (Figure1) to a suspicion of intra-pericardial collection of air. His laboratory results were all normal except for mild elevation in cardiac enzymes. Echocardiography showed normal study, the suspicion of the intra-pericardial air collection had led us to do a chest computed tomography (CT) (Figure 2) which revealed the presence of air inside the pericardium. The patient was still in intractable pain with serial elevating cardiac enzymes so we decided to do coronary angiography that revealed normal coronaries (Figure 3).
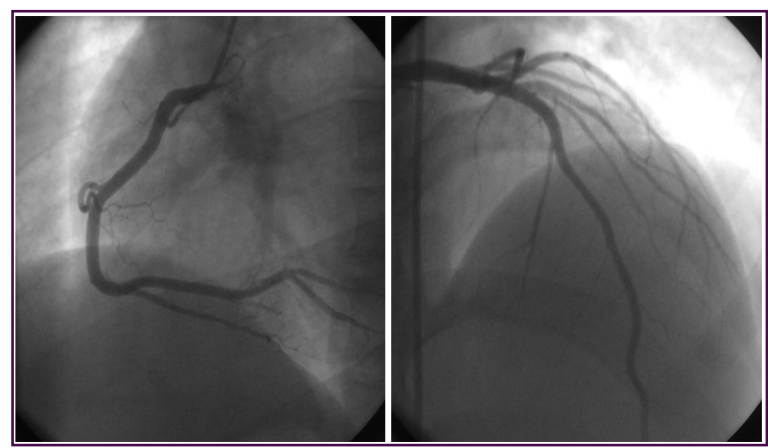

Figure 3: Invasive coronary angiography showing normal epicardial coronary arteries with dominant right system.

Patient was treated conservatively and the pain subsided few days later.

\section{Discussion}

Pneumopericardium was first described by Bricheteau in 1844 who named the sign of bruit de Moulin (water wheel sound) associated with Pneumopericardium [2]. Pneumopericardium occurs typically after the affection of the pericardium by either traumatic or non-traumatic causes. In adults, $60 \%$ of pneumopericardium results from trauma either sharp or blunt. Other causes attributed to pneumopericardium can be non-iatrogenic or iatrogenic [3]. Pneumopericardium may be symptomatic or asymptomatic depending on the quantity of air 
in the pericardium. The patient with a small pneumopericardium may be asymptomatic and the cardiac examination may be normal. In large pneumopericardium the patient may be symptomatic and the note may be tympanic and heart sounds may be metallic. The commonest symptoms are dyspnea and precordial chest pain [2].

Unlike pneumothorax, pneumopericardium often does not require any specific treatment and is usually self-limiting as in the present case. Treatment is may be only required in cases of large and symptomatic pneumopericardium or in patients of tension pneumopericardium, which can be a needle aspiration or tube decompression. Oxygen therapy in high concentrations as in management of pneumothorax can also be helpful in absorption of air [1].

\section{Conclusion}

This case demonstrates the importance of good interpretation of the chest X-ray for exclusion of non-coronary causes of chest pain.

\section{References}

1. Chopra V, Garg N, Mrigpuri P (2013) Spontaneous pneumopericardium an unusual complication in a patient of HIV and pulmonary tuberculosis. Lung India 30(2): 148-150.

2. Arda K, Eldem O, Sigirci A, Ozkan FO (2000) Spontaneous pneumopericardium and pneumomediastinum. Asian Cardiovasc Thorac Ann 8: 59-61.

3. Katzir D, Klinovsky E, Kent V, Shucri A, Gilboa Y (1989) Spontaneous pneumopericardium: Case report and review of the literature. Cardiology 76(4): 305-308.

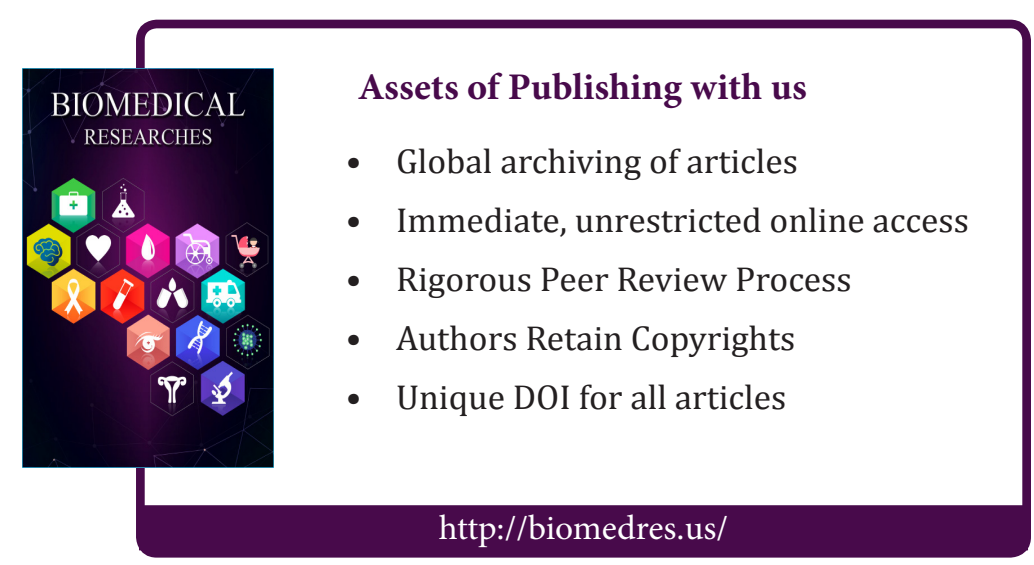

